\title{
Los gabinetes de comunicación on line de las empresas del Ibex 35
}

\author{
Berta García Orosa \\ Universidad de Santiago de Compostela \\ bertago@gmail.com
}

\begin{abstract}
Resumen
Las principales empresas españolas potenciaron en los últimos años sus salas de prensa on line con un paulatino avance hacia la comunicación 2.0. El siguiente artículo analiza la adaptación a la red de las entidades del IBEX 35. La tendencia señala una preocupación por la comunicación con los nuevos públicos, un incremento de los recursos hipertextuales y multimedia pero una menor aproximación a la conversación real con los públicos en internet.
\end{abstract}

Palabras clave: gabinetes-sala de prensa on line- comunicación organizacional-relaciones públicas-TIC

\section{Online press rooms in the Ibex 35 companies}

\begin{abstract}
Online press rooms of the most import spanish companies grow during the last year. This article discusses the adaptation to internet Ibex 35 entities. The conclusion indicates a concern for the communicating with new audiences, an increase of the hypertext and multimedia resource, but a lower approximation to the actual converse with the public.
\end{abstract}

Key words: online press room-organizational communication- public relations-ICT

\section{Referencia normalizada:}

García Orosa, B. (2013) Los gabinetes de comunicación online de las empresas del Ibex 35. Historia y Comunicación Social. Vol. 18 Nº Especial Octubre. Págs. 295-306

Sumario del artículo: 1. Introducción. 2. Metodología. 3. Los gabinetes de comunicación en internet. 4. Un nuevo modelo de comunicación. 5. Conclusiones. 6. Bibliografía.

\section{Introducción}

Durante las últimas décadas en España, los gabinetes de comunicación empresariales influyeron no sólo en el devenir de la comunicación organizacional sino, en gran medida, en el avance del periodismo en la red a través de su influencia en la agenda mediática. Las empresas españolas fueron pioneras en la comunicación organizacional en nuestro país y en el despegue de las salas de prensa on line. En la actualidad, la gestión del entorno digital es uno de los principales temas estratégicos según se recoge en el European Communication Monitor (ECM-2011). En España, casi 7 de cada 10 directores de comunicación, cree que el principal reto al que debe hacer frente la disciplina tiene que ver con dominar la evolución digital y la web 
social (Informe de Dircom, 2012: 32), preocupación que ha subido 30 puntos en los últimos cinco años.

En este artículo se realiza una radiografía de la situación de los gabinetes on line de las empresas del Ibex 35. El trabajo se enmarca en la teoría de la comunicación organizacional que, en España, se desarrolló fundamentalmente a partir de los trabajos de la década de los 90 del pasado siglo y principios del presente, aunque no podemos olvidar que el primer libro español sobre la materia fue publicado por Luis Marqués Carbó y Luis Marqués Canos en 1957 bajo el título "Las Relaciones Públicas en el ámbito local". A través de estas aportaciones se constituyó la historia de los gabinetes de comunicación cuya última etapa cuenta ya con diferentes aportaciones sectoriales y sincrónicas (Paniagua-Rojano; Gómez Calderón, 2012; González Molina, 2011; Cantalapiedra, 2012: Durántez Stolle, 2011, Túñez, Sixto, 2011 o el de la propia autora en 2009 entre otros), a la que se une la del siguiente artículo.

\section{Metodología}

Los resultados presentados en esta comunicación son fruto de un estudio exploratorio y descriptivo con el objetivo fundamental de estudiar la última etapa de la historia de los gabinetes empresariales en España. La hipótesis principal señala el gran desarrollo de las salas de prensa on line y su adaptación a la hipertextualidad y multimedialidad pero, en menor medida, a la interactividad y actualización.

La muestra analizada está constituída por las empresas incluidas en el Ibex 35 que en el período estudiado son las siguientes: Abertis SE.A, Acciona, Acerinox, ACS, Amadeus, Arcelormit, BA Popular, BA Sabadell, BA Santander, Bankinter, BBVA, BME, Caixabank, Dia, Ebro, Foods, Enagas, Endesa, FCC, Ferrovial, Gas Natural, Grifols CL.A, IAG, Iberdrola, Inditex, Indra A, Jazztel, Mapfre, Mediaset, OHL. R.E., Repsol, Sacyr, TEC. Reunidas, Telefónica y Viscofan. Dentro de la web de cada una de ellas se trabajó el apartado destinado a la relación con los medios de comunicación denominados habitualmente "sala de prensa". La técnica utilizada para la recogida de datos fue el análisis de contenido de las páginas de las principales empresas en España. Se trabajó con los siguientes ítems que pretendían responder a las preguntas de la investigación desde una perspectiva histórica:

1. Presencia de la sala de prensa dentro de la web de la empresa.

2. Importancia de la sala de prensa dentro de la página.

3. Evolución del modelo de comunicación CCCA (contenido, conversación, conocimiento y actualización).

4. Transformación de los principales instrumentos de información y comunicación.

5. Uso dado a las herramientas de trabajo en la red. 


\section{Los gabinetes de comunicación en internet}

El inicio de la primera actividad de canalización de información desde diferentes entidades de la sociedad hacia los medios de comunicación es difuso porque desde siempre, los sectores hegemónicos de la sociedad aparecen ligados a los medios de comunicación y, de alguna forma, intentaron controlarlos (García Orosa, 2005: 51). El origen de los gabinetes de comunicación podría remontarse a la Revolución Industrial o a la figura de Napoleón con su Oficina de la Opinión Pública (Parckard, Vance, 1967), a la del canciller Bulow, quien formalizó una oficina para relacionarse con los periodistas (Weil, 1992), o, desde una perspectiva más estricta, a las últimas décadas. Aunque con historias diferentes, los gabinetes en Estados Unidos y en Europa tuvieron un origen y desarrollo distintos (Rodríguez Salcedo, 2012: 334) pero con gran influencia siempre en los temas de debate públicos.

En España, los límites temporales del inicio de los gabinetes de comunicación son imprecisos. Autores como Carlos Sotelo ubican la primera experiencia de relaciones públicas en la década de los 60 vinculada a la actividad publicitaria y al servicio de entidades apolíticas. La primera agencia de RRPP se fundó en Barcelona en 1960 con el nombre de SAE de Relaciones Públicas bajo la dirección de Joaquín Mestre y Joan Viñas, mientras que un año más tarde se creaba en Barcelona la Asociación Técnica de Relaciones Públicas (García Orosa, 2005: 60). Antonio Castillo (2004: 56-57) señala cómo se desarrolla a partir de la década de 1945 con una gran influencia de Estados Unidos: $<$ La actividad de relaciones públicas llega a España a raíz de la implantación de empresas multinacionales que trasladan su estructura interna y su funcionamiento. Eso creó una creciente mimetización de esas rutinas en algunas empresas españolas>. Martín Martín (1998: 37) puntualiza que es a finales de los sesenta y comienzo de los setenta cuando algunas empresas privadas, principalmente financieras, comienzan a <abrirse hacia los medios de comunicación $>$.

Con estas experiencias previas, podemos señalar la transición de la dictadura a la democracia y, sobre todo, la década de los 80 del pasado siglo como los dos momentos de despegue de los gabinetes de comunicación en todos los sectores y, de modo particular, en el mundo empresarial. Poco a poco, las empresas pasaron del oscurantismo y el silencio sobre su gestión a la imprescindible comunicación de la misma. Primero a través de los departamentos de marketing y de publicidad y, paulatinamente, con la creación de gabinetes de comunicación responsables de la gestión integral de la comunicación, intentan crear una imagen positiva sobre su entidad ante sus diferentes públicos. Cada vez más vinculados a la dirección de las empresas, los directores de comunicación fueron asumiendo responsabilidades y nuevos retos entre los que se encuentra la comunicación con unos públicos aparentemente más activos gracias a la irrupción de las redes sociales en los últimos años.

Sin duda, desde el año 2000, el correcto empleo de las TIC se destapa como uno de los principales objetivos de los gabinetes de comunicación. Sin embargo, la implementación de una estrategia específica para la red dentro del plan de comunicación y la creación de espacio en la web fue paulatina como indican los diferentes estudios 
e informes sectoriales realizados en España en las últimas décadas. En este sentido, Ana Almansa, señala en 2004 (p. 13) que < los gabinetes de comunicación no utilizan todo lo que pudieran las ventajas que supone internet. Aunque algunos suelen enviar información por e-mail, aún mantienen en paralelo el uso del fax. Igualmente, no se suele facilitar imagen ni sonido a través de la red (aunque existen aún algunas deficiencias tecnológicas, hoy por hoy, internet permite la divulgación de imagen y sonido en unas condiciones aceptables, lo que debería traducirse en un mayor uso) $>$. Según una encuesta realizada por Dircom hace cinco años (2008), más del 66\% de los directores de comunicación manifiesta tener un interés alto o muy alto en las nuevas tecnologías pero, al mismo tiempo, más del $55 \%$ se considera un usuario intermedio, y sólo el $28 \%$ cree tener un nivel alto o muy alto como usuario de las herramientas de comunicación más novedosas. En la actualidad se indica que la revolución digital es (y seguirá siendo en los próximos años) un factor determinante en la evolución del director de comunicación (VVAA, 2013: 14) y que el dircom es la figura que ejerce (y ejercerá) el control sobre los nuevos canales online, "vehículos esenciales para la difusión de la marca según indica Maite Alba, directora de Marketing y Comunicación de Accenture (VVAA, 2013: 12) . En este contexto, la presencia y actividad de la empresa en la web constituye uno de los activos más importantes tal y como nos desvela la práctica y los propios informes elaborados por profesionales y científicos. En las próximas líneas veremos la situación actual de las salas de prensa on line de las empresas del Ibex 35.

\section{Un nuevo modelo de comunicación}

Casi cuatro de cada 10 empresas (el 37,14\% de las analizadas) empiezan a crear su identidad corportiva en la red antes de 2003. En 2008 la práctica totalidad de los departamentos de comunicación de las principales entidades en España tenían página web y correo electrónico que utilizaban diariamente en su relación con los medios de comunicación. El porcentaje en la infraestructura se reducía un poco en las entidades de menores dimensiones: $73 \%$ con internet y el 64,28\% utilizaba habitualmente el correo electrónico, pero sin modificaciones en la estructura ni en la dinámica de trabajo (García Orosa, 2009: 22). En la actualidad, en el caso de las empresas del IBEX 35, ocho de cada diez tiene una sala de prensa on line en su espacio web (de estas, el $60 \%$ en primer lugar), un $8,5 \%$ no tiene y $11,42 \%$ cuenta con un apartado de "Noticias".

Entre 2008 y la actualidad, detectamos algunos cambios formales y de contenido importantes. En el primer ámbito, se registra un avance en la unificación de la denominación, contenido y funciones del espacio objeto de estudio. "Sala de prensa" es el nombre que triunfa en el ámbito empresarial español. En segundo ámbito, una apuesta por la adaptación de las características de la red y un aparente intento de comunicación con sus públicos. Hace un lustro, el 98,7\% pertenecían a los denominados gabinetes 1.0 o gabinete básico con transmisión de información unidireccional y asimétrica y sólo el $1,3 \%$ se aproximaba al gabinete 2.0 permitiendo al receptor 
difundir y jerarquizar el mensaje en diferentes lugares de la web pero nunca modificarlo. En la actualidad, continúan primando el empleo de instrumentos de estas características pero, como indicábamos con una importante evolución hacia la 2.0. Analicemos brevemente los instrumentos de comunicación que podemos utilizar que se distribuyen en el esquema de cuatro puntos: crear mensaje, actualizar contenidos, conocer e investigar y conversar (adaptado de García Orosa: 2009).

Pese a que la filosofía del transmisor de información unidireccional y asimétrica continúa subyacente en muchos de los mensajes lanzados por los gabinetes de comunicación, en todos los ámbitos se registran avances importantes aunque no en todos los casos impliquen transformaciones relevantes en el modelo de comunicación. A continuación ofrecemos un resumen de los mismos.

En relación con el primer punto, la creación de mensajes, el avance de los gabinetes de comunicación en los últimos años fue importante en varias direcciones. En primer lugar, en el número de mensajes y en la adaptación de los mismos a la red. Como podemos observar en las imágenes 1 y 2 , las notas de prensa y noticias ofrecidas por los gabinetes de comunicación se adaptaron a las características de la red, fundamentalmente a la hipertextualidad y multimedialidad y, en menor medida, a la actualización y la interacción con el usuario. El hipertexto, la unión con temas externos al comunicado y las etiquetas permiten la creación de un nuevo discurso para la entidad.

Imagen 1. Evolución de las notas en Repsol.

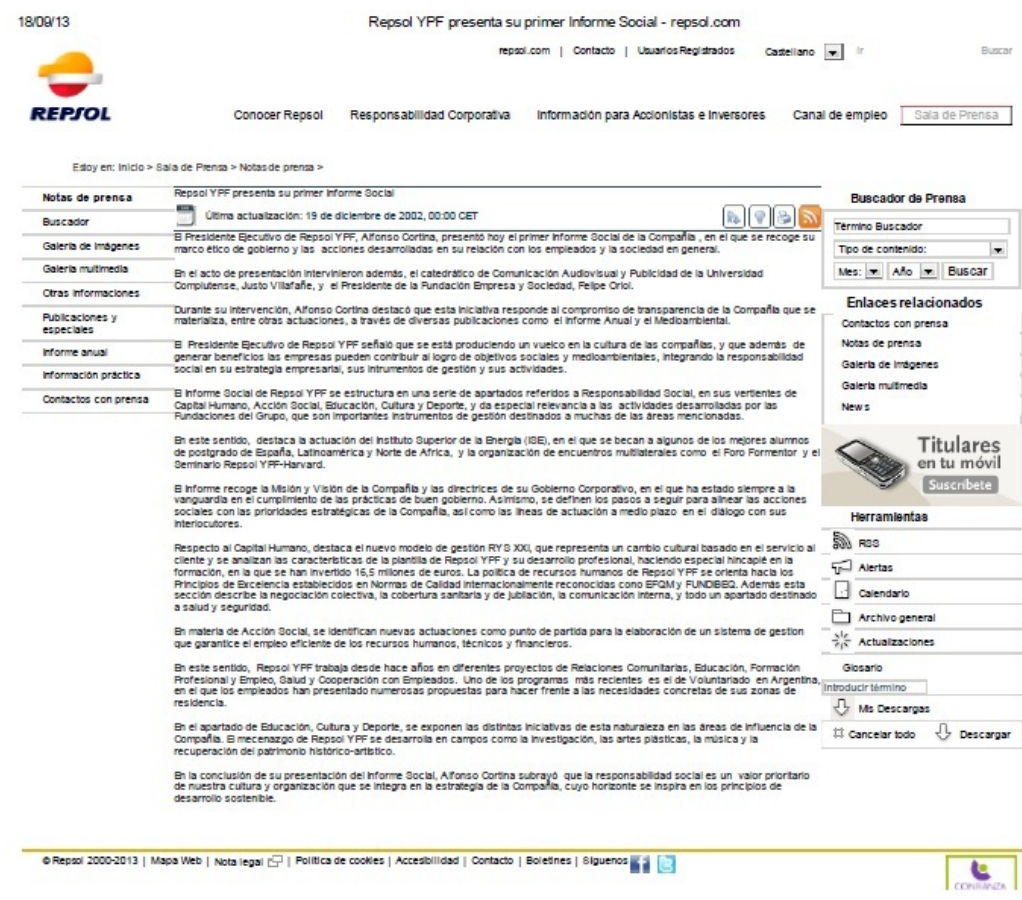




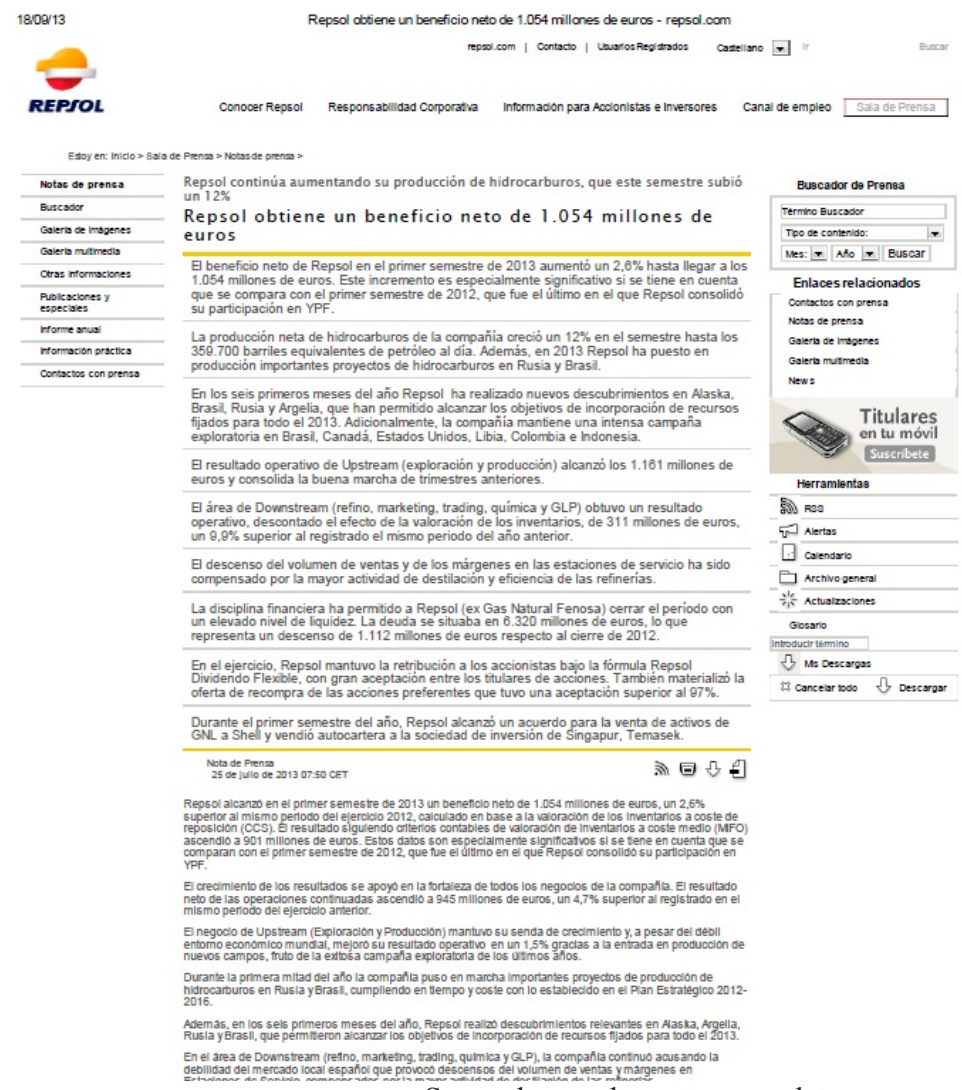

Se pueden ver las notas completas en: www. Repsol.com

Imagen 2. Evolución de las notas en Ferrovial.

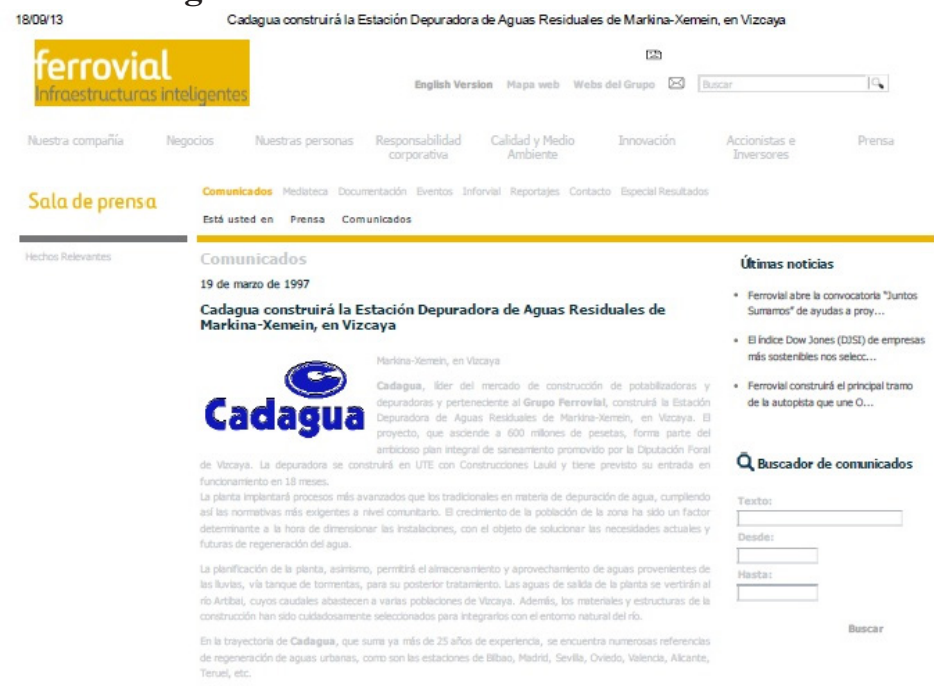




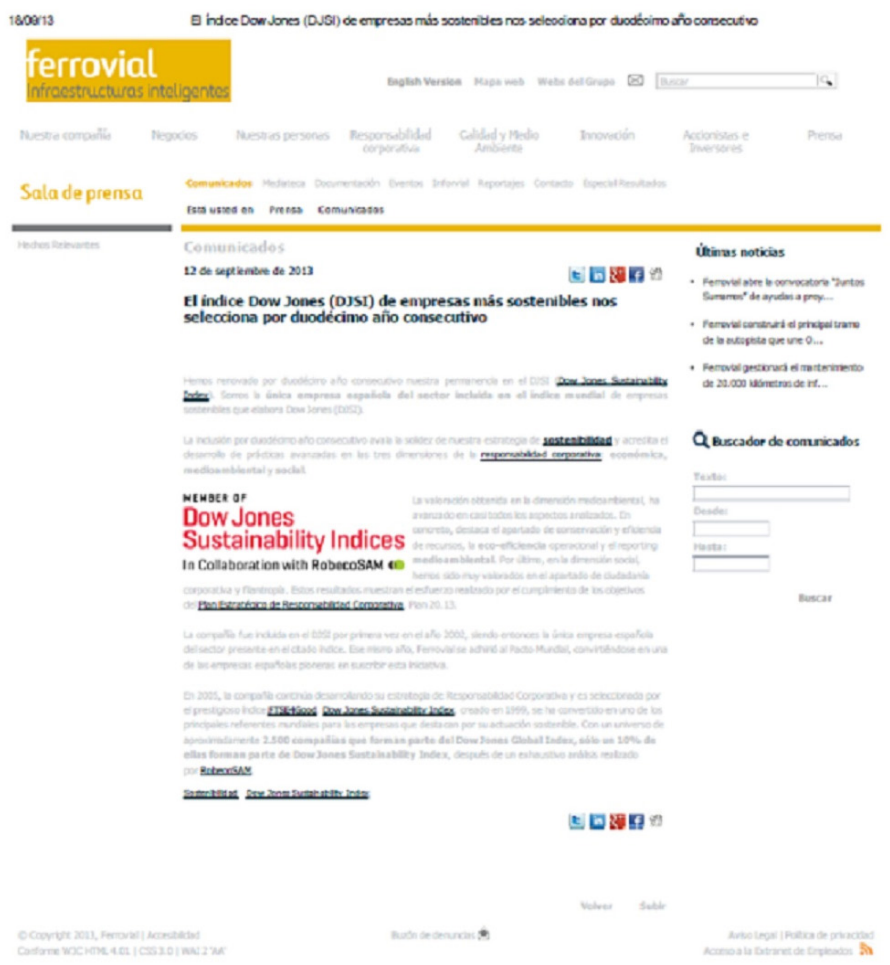

Se pueden ver las notas completas en: www. ferrovial.com

Los instrumentos utilizados en la sala de prensa on line continúan la tendencia marcada desde un inicio, con un claro predominio de los apartados dedicados a la transmisión de información de modo unidireccional y asimétrico: desde la empresa a los medios de comunicación. En la siguiente tabla se puede observar cómo las noticias, los comunicados de prensa y la documentación continúan teniendo un lugar privilegiado como ocurría en los últimos años pero se unen al incremento de otros productos como los kit de prensa, diccionarios, glosarios y reportajes.

Tabla 1. Principales instrumentos de las salas de prensa on line de las empresas del Ibex 35. 2013 (excluidas redes sociales).

\begin{tabular}{|l|c|}
\hline Instrumentos & Porcentaje de uso \\
\hline Noticias & 62,85 \\
\hline Notas/comunicados/convocatorias & 100 \\
\hline Multimedia & 100 \\
\hline Documentación & 88,57 \\
\hline Revista & 15 \\
\hline Identidad corporativa & 39,39 \\
\hline Agenda & 24,24 \\
\hline
\end{tabular}


En este sentido, la gran evolución se detecta en los dossieres de prensa y en los documentos audiovisuales (más que sonoros) que no se prodigaba en la década anterior (Del Castillo, 2008: 17). Como incremento destacado, podemos señalar el multimedia que si bien hace cinco años simplemente asomaba la cabeza por algunas páginas, hoy tiene un apartado destacado en cada una de ellas que suele llevar la denominación de "multimedia" y englobar diferentes aspectos protagonizados por las fotografías institucionales de edificios y directivos y por la oferta de diferentes formatos de los mismos documentos.

Otros avances importantes se registran en la estrategia de elaboración del propio mensaje. Si al principio las salas de prensa eran repositorios de los documentos construidos - y a veces utilizados- en entornos off line y, por lo tanto, con sus características, observamos que cada vez más intentan adaptarse a la red en cuanto a hipertextualidad y multimedialidad pero, sobre todo, en cuanto a estructura del propio mensaje. De este modo, el dircom ya no sólo "cuelga" documentos sino que los jerarquiza en función de una determinada estrategia de comunicación que le permita llegar rápidamente al usuario. Así, se incluyen buscadores, tags de la sala de prensa y apartados que nos destacan aquello que más puede interesar a la empresa bajo el título de "lo más leído, lo más buscado" como reflejo, a la vez del feed-back con el usuario.

En este apartado, debemos destacar el escaso pero con evolución ascendente uso de la geolocalización. Los instrumentos de geoubicación engloban un gran potencial como herramientas para la transmisión de información o para su difusión en los medios pero son escasamente utilizados por las salas de prensa on line empresariales en la actualidad.

La actualización de contenidos y el conocimiento de los públicos, son dos de los puntos más trabajados por los directores de comunicación desde el inicio pero solamente se adaptaron a los avances tecnológicos en los últimos años con especial interés por la transmisión a dispositivos móviles como el caso de Repsol y el envío de titulares. La actualización es un tema pendiente de las salas de prensa que continúan manteniendo un ritmo muy ligado a los medios tradicionales en la creación de eventos informativos, por lo tanto, de transmisión de los mismos contenidos a través de la web. Las páginas son todavía bastante estáticas y los cambios se concentran en la agenda y las notas de prensa.

El último aspecto, el de la conversación con el destinatario, es una de las claves de la web 2.0 y de las más utilizadas como imagen de progreso y modernidad por las propias entidades, ya que todas ellas hicieron en los últimos años una gran apuesta general en cuanto a las redes sociales. En 2010, las compañías del selectivo español apenas habían comenzado a incluir los medios sociales en sus estrategias de comunicación externa para aumentar su notoriedad en la red y llegar a los actuales consumidores multimedia según se recoge en el estudio "Presencia de las empresas del IBEX 35 en la web 2.0" (2010: 16). Puntualiza el estudio, que hace tres años la mayoría de las empresas del Ibex 35 sólo estaba presente en Linkedin y, las que tenían páginas en 
Facebook, las utilizaban principalmente como escaparates de sus acciones de RSC o iniciativas de patrocinio.

En un sentido similar, aparecían en 2011 los datos de VII Informe Juntas generales de accionistas de las empresas del IBEX 35 que por primera vez analizaban el uso que hacen de las redes sociales de las diferentes entidades en sus Juntas de Accionistas y concluían que comienza a crecer el uso de redes sociales como Facebook y Twitter en el entorno corporativo, pero con poco uso. Indican que se prefiere todavía crear una página específica dentro de la página corporativa para comunicar el desarrollo de la Junta a utilizar sus incipientes social media o abrir un perfil específico para comunicar los prolegómenos y marcha del evento (VII Informe Juntas generales de accionistas de las empresas del IBEX 35, 2011: 38).

Twitter y Facebook se siguen indicando como las más utilizadas en los diferentes informes realizados por entidades públicas y privadas. Uno de los últimos informes (Medialuna, 2013) señala la misma relación de redes más utilizadas pero unos porcentajes incrementados considerablemente: el $86 \%$ usa Twitter, el $71 \%$ Facebook, y Youtube un $69 \%$. Estos y otros estudios apuestan por la importancia de las redes: Además del contenido, deben incluir la posibilidad de distribución a través de otras redes como Twitter, FriendFeed, Faceboook, Menéame, Del.icio.us, etc., y otra serie de elementos imprescindibles en un nuevo entorno de Comunicación Corporativa hacia la Social Media Newsroom (Sánchez-González, María y Paniagua-Rojano, Francisco-Javier, 2013.).

En el caso de las salas de prensa on line -contabilizamos únicamente las que figuran en su entrada- la evolución es similar al conjunto de la empresa pero con porcentajes de uso mucho más reducidos, aunque con una relación de instrumentos igual. Las empresas analizadas incluyen dentro del apartado "redes sociales" los siguientes lugares: Facebook, Tuenti, Linkedin, Twitter, Youtube, Flickr. Además de estas se detectó presencia en Youtube, Slideshare, Google+, Linkedin, canales RSS, Pinterest y Tuenti (aunque no todas ellas son propiamente redes sociales optamos por respetar la propuesta de las entidades y recordamos que analizamos únicamente la sala de prensa on line). El uso de las redes sociales continúa siendo escaso dentro de la sala de prensa, objeto de estudio y descripción en este artículo. Como se puede observar en el gráfico 1, el $25,71 \%$ de las entidades analizadas tiene presencia en Twitter en la sala de prensa seguido de Linkedin con un 11,42\% y Facebook con un 2,85\%. Especial mención merece el caso de Youtube incluido en muchos casos dentro del apartado de redes sociales y que acumula un $14,28 \%$ de las entidades con un uso habitual. Se trata de una herramienta repositorio gratuita, en principio, frente a la cual muchos dircom eran reticentes por no pertenecer al plan de comunicación de la empresa pero que, con el paso del tiempo, se convirtió en una de las más utilizadas ya que no sólo implica una mayor visibilidad de la empresa sino también, en ocasiones, un mayor tráfico para la propia página web. 
Gráfico 1. Presencia en instrumentos descritos como "redes sociales". Porcentajes.

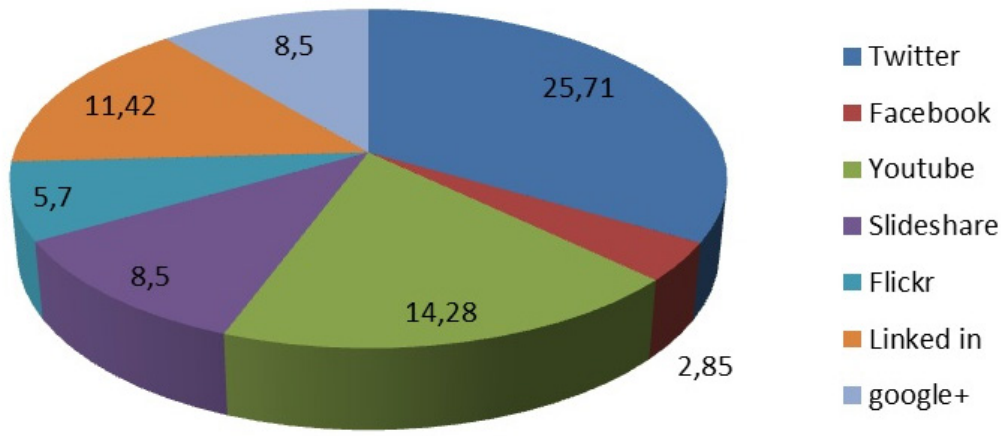

Dentro del uso habitual de las redes sociales, destaca algún caso paradigmático como el de Iberdrola que no sólo las destaca dentro de la sala de prensa, sino además incita a su uso por ejemplo, en el caso de las fotografías.

Unido a la escasa evolución de la conversación con el usuario o web 2.0, detectamos una escasa tendencia al que Fondevila Gascón (2012) denomina "de registro" e identifica como <el medio de comunicación registra la información de los usuarios. Las informaciones se adaptan (en formato y contenido) a los registros. Es una fórmula personalizada (páginas web comerciales, diarios digitales de pago)>. En el caso de las salas de prensa es una fórmula con una gran potencialidad pero todavía infrautilizada.

\section{Conclusiones}

La inversión de las entidades en las salas de prensa on line es importante y responde al espíritu recogido en los informes y observatorios de la comunicación durante las últimas décadas pero no supone, por el momento, un cambio de flujos de información ni grandes modificaciones en el discurso transmitido por la entidad.

Las características de internet y el modelo ideal de comunicación con los medios están dibujados y poco a poco las empresas españolas avanzan en algunos de ellos. La hipertextualidad y la multimedialidad registran grandes avances en los últimos años pero permanece como materia en desarrollo la comunicación con los públicos. 


\section{Referencias bibliográficas}

ALMANSA MARTÍNEZ, Ana (2004): "Historia de los gabinetes de comunicación en España”. En: Revista Historia y Comunicación Social, n ${ }^{\circ}$ 9. Madrid: p.5-21.

CASTILLO ESPARCIA, Antonio (2004): "Investigación sobre la evolución histórica de las relaciones públicas". En: Revista Historia y Comunicación Social, nº 9. Madrid: p.43-62.

FONDEVILA GASCÓN, Joan Francesc (2012). "El uso de recursos del periodismo digital en la prensa del Reino Unido, Francia, Estados Unidos y España". En: Estudios sobre el Mensaje Periodístico, Vol. 18, Núm. 1. Madrid: p. 73-87.

GARCÍA OROSA, Berta (2005): Los altavoces de la actualidad. Radiografía de los gabinetes de comunicación. A Coruña: Netbiblo.

GARCÍA OROSA, Berta (2009): Gabinetes de comunicación on line. Claves para generar información corporativa en la red. Sevilla: Comunicación social.

MARTÍN MARTÍN, Fernando (1998): Comunicación empresarial e institucional. Editorial Universitas. Madrid.

MARTIN MARTIN, Fernando (1999): Comunicación empresarial e institucional. Madrid: Editorial Universitas.

MEDIALUNA (2013): “¿Cómo comunican las empresas más relevantes del país en redes sociales?", disponible en http://es.slideshare.net/Medialunacom/observatorio-redes-sociales-empresas-del-ibex-35-25848221Medialuna [26-09-2013]

NOGUERO, A. \& PELÁEZ SÁNCHEZ, I. (2013): “The evolution of Dircom: from communication manager to reputation strategist". En: Communication\&Society/ Comunicación y Sociedad, Vol. 26, n. 1, 2013. Pamplona: p. 47-66.

PARCKARD, Vance (1967): Las formas ocultas de la propaganda. Buenos Aires: de.Edsudamericana.

RODRÍGUEZ SALCEDO, Natalia (2012): "Mapping public relations in Europe: writing national histories against the US paradigm". En Comunicación y sociedad, vol XXV, n. 2. Pamplona: p.331-373.

SÁNCHEZ-GONZÁLEZ, María; PANIAGUA-ROJANO, Francisco-Javier (2013). "Estrategias de comunicación 2.0 en asociaciones profesionales. Estudio del caso de los Colegios Oficiales de Médicos en España”. En: Revista Mediterránea de Comunicación, vol. 4, n. ${ }^{\circ} 1$. Alicante: 21-51.

TÚÑEZ, Miguel; SIXTO, José (2011): "Redes sociales y compromiso 2.9: la comunicación de los diputados españoles en facebook". En: Revista Latina de Comunicación Social, n- 66. La Lagura: p 1-25.

VVAA (2010): Presencia de las empresas del IBEX 35 en la web 2.0, disponible en Www.estudiodecomunicacion.com, p. 16 [26-09-2013].

VVAA (2011): VII Informe Juntas generales de accionistas 2011. Empresas del IBEX 35. Foro de Buen Gobierno y Accionariado, IESE.

VVAA (2012): Anuario de la comunicación Dircom. Madrid: Dircom.

VVAA (2013). El Dircom del futuro y el futuro del Dircom. Madrid: Burson-Marsteller.

WEIL, Pascale (1992): La comunicación global: comunicación institucional y de gestión. Barcelona: Paidós. 


\section{La autora}

Licenciada en Ciencias de la Información, en Ciencias Políticas y de la Administración y Doctora en Periodismo por la Universidad de Santiago de Compostela (USC). Actualmente es profesora titular en la Facultad de Comunicación de la USC. Es miembro del grupo de investigación Novos Medios desde su creación y ha publicado varios artículos y libros sobre comunicación organizacional, periodismo y ciberperiodismo. Entre sus últimas publicaciones están Gabinetes de comunicación on line. Cómo generar información corporativa en la red (Comunicación Social, 2009) o "La conformación de valores noticia en un contexto de conflicto. Análisis pragmático de su funcionamiento en la información sobre el narcotráfico en México y el feminicidio en Ciudad Juárez", en (Estudios del Mensaje Periodístico, diciembre 2012). 